\title{
Cathodoluminescence Study of Actinide Oxides
}

\author{
C.C. Davis, ${ }^{*}$ A.D. Neuman, ${ }^{*}$ T.A. Nothwang, ${ }^{*}$ W.S. Duncan*, F.G. Hampel*, and S.P. Wilson** \\ * Materials Science and Technology Division, Los Alamos National Laboratory, M.S. E574, Los \\ Alamos, NM 87545 \\ ** Plutonium Manufacturing and Technology Division, Los Alamos National Laboratory, M.S. \\ E505, Los Alamos, NM 87545
}

In this study, actinide oxides (plutonium, uranium, neptunium, and americium oxides) were imaged to enable characterization of their morphology, particle size, cathodoluminescence (CL) signal, and trace elemental content using a scanning electron microscope equipped with a cathodoluminescence detector. Unexpectedly, neptunium oxide $\left(\mathrm{NpO}_{2}\right)$ powder was found to have two unique CL signals [1-3]. The $\mathrm{NpO}_{2}$ powder was analyzed via energy dispersive spectrometry to identify which elements were present with the hope of determining the origin of the two CL signals. This analysis determined that magnesium $(\mathrm{Mg})$, chlorine, and calcium $(\mathrm{Ca})$ were the elements that caused the multiple CL signals. These samples were also analyzed in the electron microprobe. While undergoing electron probe microanalysis (EPMA), some of the particles could be seen fluorescing in the optical microscope on the microprobe, see Figure 1. An attempt was made to capture an image of the fluorescing $\mathrm{NpO}_{2}$ particle prior to determining the distribution of neptunium via wavelength dispersive spectroscopy. This particle was damaged by the beam after a short time, see Figure 2 .

These initial studies have resulted in the need to expand the research project and develop the use of CL element distribution imaging. Since some materials emit CL signals, i.e. characteristic secondary electron excitation in the visual light spectrum, it is expected that CL can be used as another technique to identify trace elements [4]. Based on the initial study, it is anticipated that CL imaging will be faster than traditional EPMA mapping. However, to ensure that the CL signals have been correctly identified, the next analysis will be verification of the CL signals using elemental standards. Once the CL signals are verified, CL imaging of fuel pellets can be used to reveal the distribution of the actinide elements as well as the non-actinide elements. These analyses will confirm that CL imaging can be a viable tool and provide complementary data to supplement traditional mapping techniques.

References

[1] A.D. Neuman, C.C. Davis, T.A. Nothwang, F.G. Hampel, S.L. Voit, M.R. Lopez, and A.C. Martinez, "Characterization of Minor Actinide Mixed Oxide Fuel," Journal of Nuclear Materials, Volume 385 (1), 15 March 2009, p. 168, doi: 10.1016/j.jnucmat.2008.10.021.

[2] A.D. Neuman, C.C. Davis, T.A. Nothwang, F.G. Hampel, S.L. Voit, M.R. Lopez, and A.C. Martinez, "Characterization of MA-MOX Powders and Pellets," REWAS 2008, TMS (The Minerals, Metals, And Materials Society), October 2008.

[3] T.A. Nothwang, A.D. Neuman, C.C. Davis, S.L. Voit, M.R. Lopez, and A.C. Martinez, "Particle Size and Morphology of MA-MOX Precursor Powders," REWAS 2008, TMS, October 2008.

[4] C. E. Norman, Microscopy and Analysis, March 2002, p.9-12.

[5] This work was supported by the Department of Energy (DOE)/NE-5, Global Energy Partnership, under contract DE-AC52-06NA25396. 


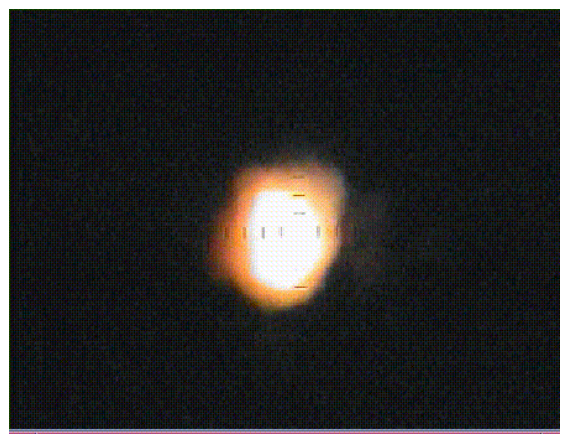

FIG.1: Optical image showing fluorescence of an impurity particle in $\mathrm{NpO}_{2}$ (NOTE: scale marker not possible due to the image's capture method. The particle is approximately $10 \mu \mathrm{m}$ in diameter).
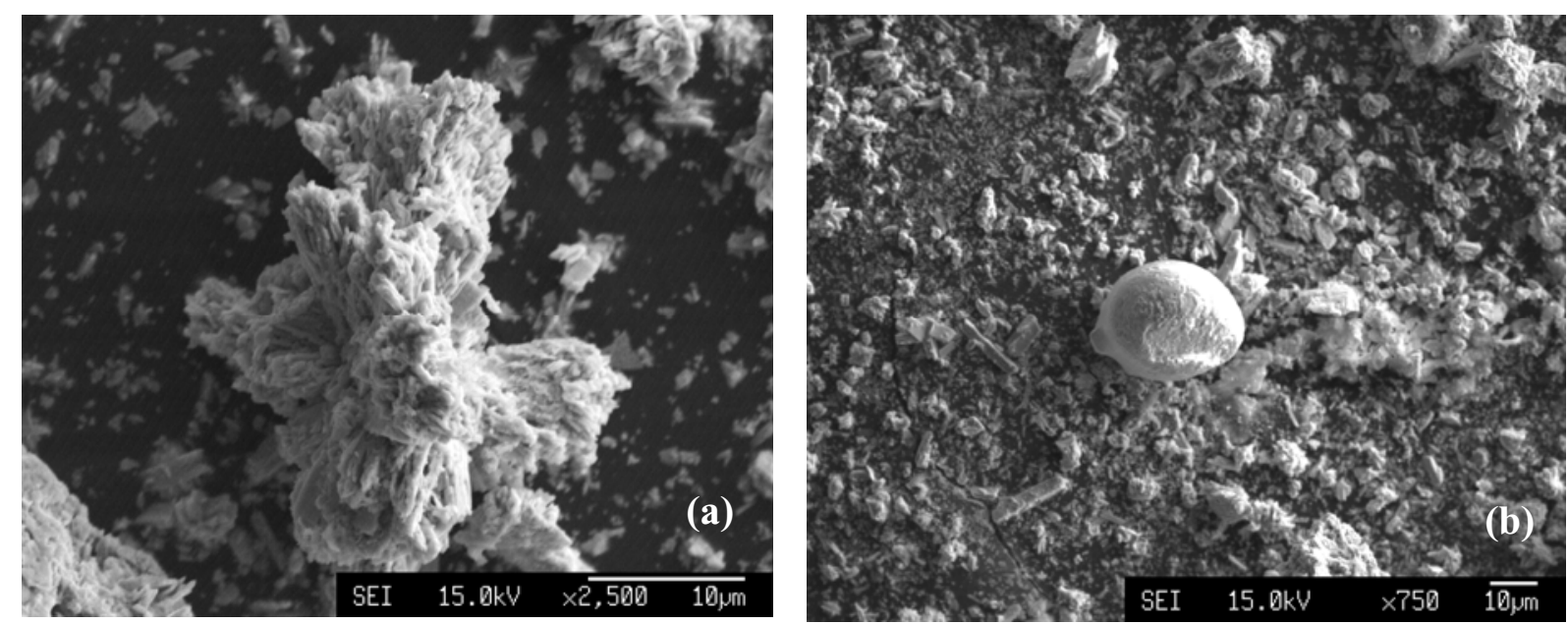

FIG 2 (a) Image of the same particle shown in Figure 1 before beam damage; (b) after beam damage.

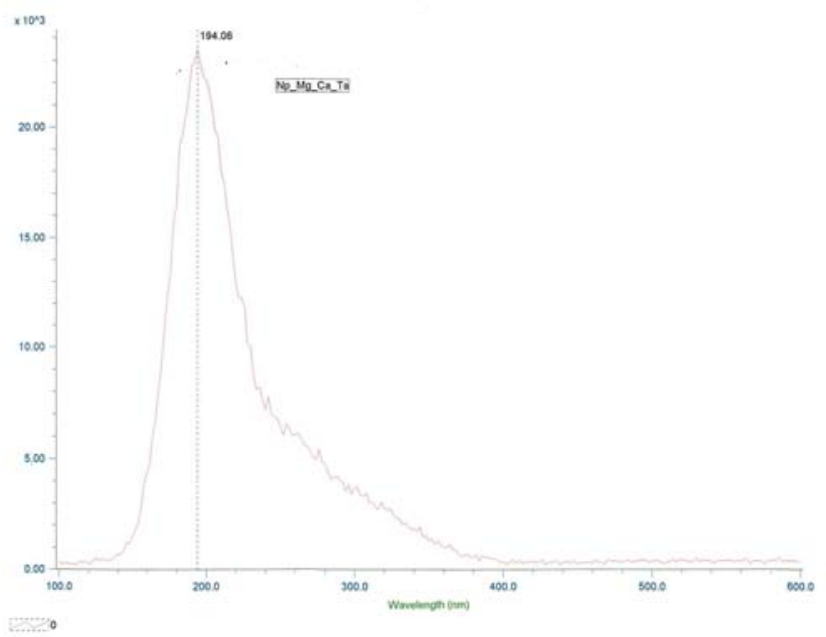

FIG 3: Cathodoluminescence linescan which reveals $\mathrm{Mg}$ and/or $\mathrm{Ca}$ impurities in $\mathrm{NpO}_{2}$. Similar analyses will be used to assess other samples. 